\title{
Challenges in plasma and laser wakefield accelerated beams diagnostic
}

A. Cianchi ${ }^{\mathrm{a}, *}$, M. P. Anania ${ }^{\mathrm{b}}$, M. Bellaveglia $^{\mathrm{b}}$, M. Castellano $^{\mathrm{b}}$, E. Chiadroni ${ }^{\mathrm{b}}$, M. Ferrario $^{\mathrm{b}}$, G. . Gatti $^{\text {b }}$, B. Marchetti ${ }^{\text {c }}$ A. Mostacci ${ }^{\mathrm{f}}$, R. Pompili ${ }^{\mathrm{b}}$, C. Ronsivalle ${ }^{\mathrm{d}}$, A.R. Rossi ${ }^{\mathrm{e}}$, L. Serafini ${ }^{\mathrm{e}}$

${ }^{a}$ University of Rome Tor Vergata and INFN, V. della Ricerca Scientifica 1, 00133 Rome, Italy

${ }^{b}$ INFN-LNF - Via E. Fermi 40, 00044 Frascati (RM), Italy

${ }^{c} D E S Y$, Platanenallee 6, D-15738 Zeuthen, Germany

${ }^{d}$ ENEA C.R. Frascati, Via E. Fermi, 4500044 Frascati (RM), Italy

${ }^{e} I N F N-M i$, Via Celoria, 1620133 Milano, Italy

${ }^{f}$ University of Rome La Sapienza, P.le Aldo Moro 5, 00185 Rome (Italy)

\begin{abstract}
The new frontier in the particle beam accelerator is the so called plasma acceleration. Using the strong electric field inside a plasma is possible to achieve accelerating gradients order of magnitude larger with respect to the actual technologies. Different schemes have been proposed and several already tested, producing beams of energy of several GeV. Mainly two approaches are followed: either the beam is directly produced by the interaction of a TW/PW class laser with a gas jet, or a preexisting particle beam is accelerated in a plasma channel. In both cases a precise determination of the emerging beam parameters is mandatory for the fine tuning of the devices. The measurement of these parameters, in particular the emittance, is not trivial, mainly due to the large energy spread and to the tight focusing of these beams or to the background noise produced in the plasma channel. We show the problems related to the diagnostic of this kind of beams and the proposed or already realized solutions.
\end{abstract}

Keywords:

beam diagnostic, plasma wakefield acceleration, emittance

\section{Introduction}

Conventional accelerating Radio-Frequency (RF) structures are limited in the achievable gradient. Depending by the used frequency it ranges between tens to one hundred $\mathrm{MeV} / \mathrm{m}$. The past few years have seen a great progress in the field of accelerating particle by means of plasma acceleration $([1],[2],[3],[4],[5],[6],[7])$. This wide category includes several different methods. We focus mainly our attention to Laser Wakefield Accelerator (LWFA) and Beam Driven Plasma Wakefield Accelerator (PWFA).

In LWFA, the ponderomotive force of a laser pulse traveling through underdense plasma can

\footnotetext{
*Corresponding author

Email address:

alessandro.cianchi@roma2.infn.it (A. Cianchi)
}

excite a plasma wave with longitudinal electric fields larger than $10 \mathrm{GV} / \mathrm{m}$. These fields may be utilized to accelerate electrons to ultrarelativistic energies on a centimeter scale.

TW-class laser are needed in order to provide the required electric field, tightly focused in a spot of few $\mu \mathrm{m}$. Therefore high divergent beams (about $1 \mathrm{mrad}$ or more) are produced, with also a big energy spread, larger than $1 \%$. While several experiments are available for such a scheme, no one has so far used the PWFA. In 2007 [8] an energy gain of more than $42 \mathrm{GeV}$ was achieved in a plasma wakefield accelerator of $85 \mathrm{~cm}$ length, driven by a $42 \mathrm{GeV}$ electron beam at the Stanford Linear Accelerator Center (SLAC). This proof of principle experiment is not exactly the PWFA. In the case of the SLAC experiment a bunch much 
longer than the plasma wavelength has been injected into the plasma, so some parts of the beam were accelerated, while other parts decelerated. The aim of the PWFA is the injection of a train of separated pulses. A plasma wave/wake is excited by relativistic particle bunches. Some of them loose energy to the plasma (driving beams), and one is accelerated (witness bunch). Typical values for plasma density are in the order of about $10^{16} \mathrm{~cm}^{-3}, 100 \mu \mathrm{m}$ for plasma wavelength and gradient in the order of $10 \mathrm{GV} / \mathrm{m}$. Bunch trains, with features that can be successfully used for this application are now available and experiment are foreseen in the next future [9].

\section{Diagnostic challenges}

So far electron beams have been produced and accelerated through plasma wakefield acceleration as proof-of-principle experiments. Now the aim is producing high quality beams. It means a severe control of the beam parameters in order to use it for several applications. The diagnostic plays a crucial role in the determination of the beam parameters. Unfortunately the conventional electron beam diagnostic faces several problems when applied to these beams. These problems will be clearly addressed in the following chapters. Moreover the main issue is the lack of simulations. The interaction of the electrons within the plasma is indeed very difficult and time consuming to simulate. Only few simulated samples of such a beams are then available. This point is crucial in order to design and realize a satisfactory diagnostic system. In this paper we focus only on the measurements of the beam emittance, that it is defined in the next chapter. This is the most relevant parameter and sometimes the most difficult to estimate. The beam current, the total charge as well as the longitudinal properties can be retrieved applying conventional diagnostic methods [10] to such beams.

\section{Emittance}

The main parameter of an electron beam is the emittance. For sake of simplicity we can define it as the volume occupied by the beam in the phase space. Under the conditions of the Liouville theorem the emittance is a motion invariant.

\subsection{Normalized Emittance}

The most common parameter in a particle accelerator is the normalized emittance. The definition of the r.m.s. (root mean square) emittance is the following:

$$
\varepsilon_{n}^{2}=\left\langle x^{2}\right\rangle\left\langle\beta^{2} \gamma^{2} x^{\prime 2}\right\rangle-\left\langle x \beta \gamma x^{\prime}\right\rangle
$$

where $\gamma$ is the usual relativistic factor, $\beta$ is the ratio between the speed of the particle and the light and $\mathrm{x}, \mathrm{x}$ ' are the position and the tranverse velocity of every particles. The average is over all the particles in the bunch. Such a definition is misleading for a typical plasma accelerated beam where both the energy spread and the angular divergence are at least an order of magnitude larger than conventional beam, as pointed out in [11]. If the correlation between the energy and transverse position is negligible, as in a drift without collective effects, we can write

$$
\varepsilon_{n}^{2}=\left\langle x^{2}\right\rangle\left\langle\beta^{2} \gamma^{2}\right\rangle\left\langle x^{\prime 2}\right\rangle-\langle\beta \gamma\rangle^{2}\left\langle x x^{\prime}\right\rangle^{2}
$$

Using the definition of energy spread

$$
\sigma_{E}^{2}=\frac{\left\langle\beta^{2} \gamma^{2}\right\rangle-\langle\beta \gamma\rangle^{2}}{\langle\gamma\rangle^{2}}
$$

and replacing it in the former equation we have

$$
\begin{aligned}
& \varepsilon_{n}^{2}=\langle\gamma\rangle^{2} \sigma_{E}^{2}\left\langle x^{2}\right\rangle\left\langle x^{\prime 2}\right\rangle+ \\
& \langle\beta \gamma\rangle^{2}\left(\left\langle x^{2}\right\rangle\left\langle x^{\prime 2}\right\rangle-\left\langle x x^{\prime}\right\rangle^{2}\right)
\end{aligned}
$$

The second term is called geometrical emittance. The first term is usually totally negligible when we consider conventional accelerated beam. In our case the large value of the energy spread as well as the big angular divergence (in the order of the mrad) makes the first term the leading one. This contribution is not constant and increases with the distance from the plasma, being the beam size mainly dominated by the angular divergence. In this scenario the normalized emittance is not constant and depends on the position of the measurement. However we focus here on the geometrical emittance, which is the measured quantity. In the [11] the relation between normalized and geometrical emittance are clearly addressed. 


\subsection{Pepper pot}

When particle beams are strongly dominated by the space charge, usually in the energy range of few $\mathrm{MeV}$, the most used technique to retrieve the emittance value is the so called pepperpot technique. Details concerning this measurement can be found in [12], while in [13] the method for data elaboration and processing is discussed.

An intercepting multi-slit or hole mask selects an array of beamlets. The space charge dominated incoming beam is thus reduced into emittance-dominated beamlets that drift up to an intercepting screen(see Fig. 1). If the screen response is linear, the intensity of beamlets spots on the screen is directly proportional to the number of particles in the beamlets which hit the screen. The emittance can be retrieved calculating the second momentum of the beam. The slits mask must stop, or largely degrade, the intercepted components of the beam, while avoiding excessive slit scattering effects.

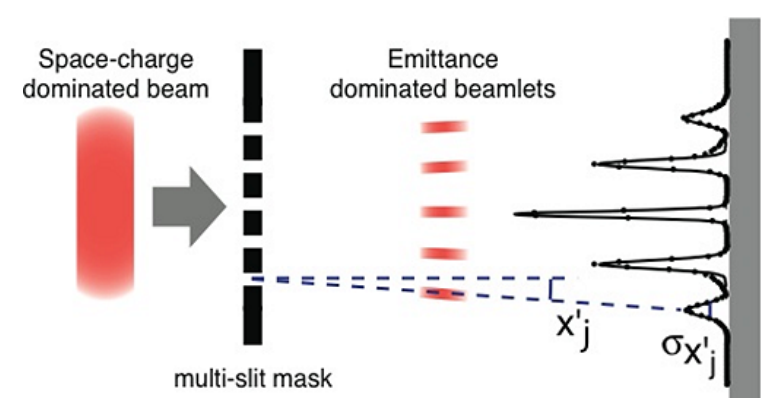

Figure 1: Pepper pot layout

Some people have already applied this technique to plasma accelerated beams, see for example [15]. However, an unwanted cut of the transverse phase space due to the used device is clearly visible in this paper. As a consequence the vertical size of the camera does not contains the whole beam. This problem leads to an underestimation of the emittance. Furthemore a critical undersampling of the transverse phase space is also present. To clarify this problem we can compare the phase space of three different situations (see Fig.2).

All of them refers to beams at $60 \mathrm{~cm}$ from the source. In A there is the phase space of a typical electron beam, $5 \mathrm{MeV}$, initial spot size $1 \mathrm{~mm}$. The pepperpot technique has been invented for
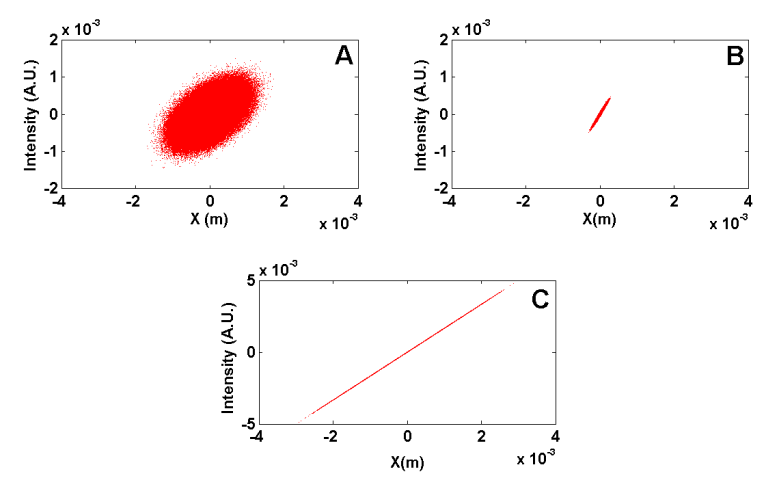

Figure 2: A: phase space of a typical electron beam, 5 $\mathrm{MeV}$, initial spot size $1 \mathrm{~mm}$, drift from the source $60 \mathrm{~cm}$; B: phase space of a possible plasma accelerated beam, 500 $\mathrm{MeV}$ initial spot size $10 \mu \mathrm{m}$, drift from the source $60 \mathrm{~cm}$; $\mathrm{C}$ : phase space of a possible beam plasma accelerated, 500 $\mathrm{MeV}$, initial spot size $1 \mu \mathrm{m}$, drift from the source $60 \mathrm{~cm}$.

this kind of beams. In B it is shown a phase space of a possible plasma accelerated beam, 500 $\mathrm{MeV}$ with initial spot size $10 \mu \mathrm{m}$. In $\mathrm{C}$ there is again a phase space of a possible plasma accelerated beam, identical to B but with initial spot size $1 \mu \mathrm{m}$. The pepperpot method samples the beam phase space. It is clear, especially from plot $\mathrm{C}$, that when we consider beams with high angular divergence with respect to the beam size, the phase space collapses in a tiny line.

In Fig.3 we compared the intensity distributions of the signal on the detecting screen downstream the mask. The number of the slits, their distance and their sizes are optimized in order to minimize the error due to the the sampling nature of this technique, considering only that values that can be used in a real tungsten slit machining [14]. We completely neglect any effect coming from the multiple scattering in the material as well as any consideration about the Signal to Noise Ratio (SNR). We focus only on the sampling properties of this method. In the plot $\mathrm{A}$ we show the distribution which results applying a mask on the beam with phase space A in Fig.2. The mask is placed at $60 \mathrm{~cm}$ and the screen at 2 $\mathrm{m}$ from the mask. The holes size is $50 \mu \mathrm{m}$, while the distance between them is $500 \mu \mathrm{m}$. In this condition the percentage error coming only from the sampling properties of this method is about $1 \%$.

On the right of Fig. 3 there is the distribution 

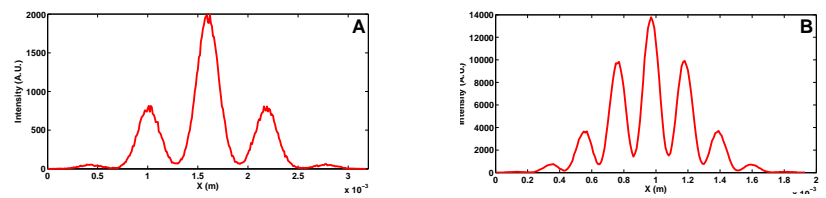

Figure 3: Left: beam initial spot size $=1 \mathrm{~mm}, 5$ holes, $50 \mu \mathrm{m}$ diameter, $500 \mu \mathrm{m}$ distance between holes, $60 \mathrm{~cm}$ from the source, $2 \mathrm{~m}$ distance between mask and screen, estimated error $=1 \%$ Right: beam initial spot size $=10 \mu \mathrm{m}$, 9 holes, $25 \mu \mathrm{m}$ diameter, $50 \mu \mathrm{m}$ distance between holes, $60 \mathrm{~cm}$ from the source, $2 \mathrm{~m}$ distance between mask and screen, estimated error $=37 \%$

of $\mathrm{B}$ type phase space. The mask is placed at 60 $\mathrm{cm}$ and the screen at $2 \mathrm{~m}$ from the mask. The holes size is $25 \mu \mathrm{m}$, while the distance between them is $50 \mu \mathrm{m}$. In this condition the percentage error coming only from the sampling properties of this method is about $37 \%$. This last beam can be representative of some type of plasma accelerated beam. An initial spot size of $10 \mu \mathrm{m}$ give an error that, even if quite larger with respect to typical emittance monitors for beam diagnostic, is still tolerable. This is not the case reported in Fig.4.

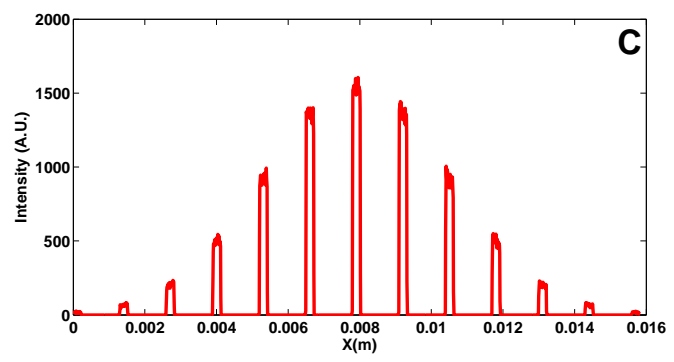

Figure 4: Beam initial spot size $=1 \mu \mathrm{m}, 13$ holes, $50 \mu \mathrm{m}$ diameter, $300 \mu \mathrm{m}$ distance between holes, $60 \mathrm{~cm}$ from the source, $2 \mathrm{~m}$ distance between mask and screen, estimated error $=1800 \%$

Here with an initial beam spot of $1 \mu \mathrm{m}$ rms, the phase space (type C Fig.2) is so thin that the sampling is completely inefficient. It is manly due to the large aspect ratio between beam size at the source and its divergence, in the order of 1:1000, while usually this value is close to $1: 1$ !

Reference [16] reports on an interesting measurement of the beam emittance using this technique. Except for the definition of the normalized emittance, that we have already shown is improper in this case, a simple estimation of the undersampling error in their case leads to a reasonable $47 \%$ in the case of an initial spot size of $10 \mu \mathrm{m}$ and a value 20 times larger if we suppose it was about $1 \mu \mathrm{m}$. Therefore we can conclude from our analysis that, if we consider the error due to undersampling the thin phase space the main limiting factor of this measurement, a initial beam spot size less than $10 \mu \mathrm{m}$ rms produces a phase space that cannot be resolved with this technique. The problems related to the multiple scattering as well as the SNR ratio in case of high energy beams does not seems a limiting factor as clearly addressed in [17].

\subsection{Quadrupole scan}

The main advantage of the pepperpot technique is the single shot capability. The intrinsic instability inside the plasma channel produces different beams from shot to shot. If we can consider to accumulate a long statistics in order to damp these fluctuations also multi shot measurements are feasible. In this scenario the most popular technique for emittance measurement is the quadrupole scan [10]. It is possible to measure the beam emittance changing the strength of a magnetic lens and recording the beam spot size downstream. With at least 3 different measurements is possible to retrieve the elements of the sigma matrix that are related with the emittance. The main concern is the large energy spread of the beam that produces an unwanted emittance dilution due to chromatic effect in the quadrupoles. We evaluated [18] which is the contribution of the chromaticity in an emittance measurement with a single quadrupole. A moderate spot size, about $300 \mu \mathrm{m}$, with an energy of the order of $150 \mathrm{MeV}$, an energy spread of about $1 \%$ and a normalized emittance of $1.5 \mathrm{~mm}$-mrad, gives an emittance dilution only of $1 \%$. But if the spot size is increased up to $1.7 \mathrm{~mm}$ for instance, the increment in the emittance is already in the order of $50 \%$.

Due to the large angular divergence of the beam the spot size is almost completely dominated by the divergence. So the distance between the beam waist and the quadrupole used for the diagnostic must be set so that the beam size is so small at 
that energy that the chromatic effects do not affect the measurement. For instance in the case considered above half a meter is a reasonable distance. This value must be compatible with the experimental setup. Usually the plasma interaction happens in a dedicated vacuum chamber and sometimes mechanical constrains avoid to place any device at a distance shorter than $10-20 \mathrm{~cm}$. Therefore we consider the quadrupole scan technique, even if with the problems that we highlighted concerning the multi-shot nature of such a diagnostic and the limits that can arise for mechanical constrains, a promising candidate for reliable emittance measurement.

\subsection{Multi monitors}

Another popular method for emittance measurement is the so called multiple monitors. In this case the beam size is measured in three different positions and the values of the beam spots are used in order to retrieve the emittance. This is a multi shot measurement. A very intriguing experiment is presented in [19]. The authors used very thin aluminum foils (about $3 \mu \mathrm{m}$ thickness) to produce optical transition radiation when the beam pass through the foils. The radiation coming from every screen is collected by 3 different CCD camera at the same time. Therefore this device can be used to produce single shot measurement. The contribution of multiple scattering to the intrinsic angular spread of the beam is demonstrated to be negligible in their experimental conditions. They tested the system on a conventional accelerated electron beam at $3 \mathrm{GeV}$. The multiple scattering angle is inversely proportional to the particle energy. When we consider to apply this method to beams of energy ten times smaller, the contribution of multiple scattering is not negligible anymore. For this reason we consider the method very interesting but only for beam energies greater than $1 \mathrm{GeV}$.

\section{Conclusions}

In this paper we focused on the difficulties related to the measurement of the emittance of particle beams accelerated by means of plasma interaction. The main problem of these beams are the big energy spread, larger than $1 \%$, and the huge angular divergence, more than $1 \mathrm{mrad}$. We pointed out that in this condition the usual definition of normalized emittance must be reconsidered. We also compared several method in order to measure the emittance. We conclude that single shot methods are preferable, due to the large instability in the plasma acceleration process. In this scenario we evaluated that pepperpot systems cannot be used when the initial beam size is smaller than $10 \mu \mathrm{m}$, because the tiny phase space is strongly undersampled.

The multiple screen method could be considered also as a good alternative, using thin foils to produce optical transition radiation and imaging at the same time to have a single shot measurement. However the increasing of the emittance due to multiple scattering set a lower limit of the beam energy where it is possible to use this method, above $1 \mathrm{GeV}$. In case of multishot measurements, if we can accumulate enough statistic to dump the fluctuations, we demonstrated that also the quadrupole scan technique is feasible. In this case the main constrain is the beam size, that being closely related to the angular divergence set an upper limit to the distance between the magnetic lens and the plasma channel.

\section{References}

[1] Faure et al. Nature 431, 541 (2004)

[2] Mangles et al. Nature 431, 535 (2004)

[3] Geddes et al. Nature 431, 538 (2004)

[4] Hidding et al. Phys. Rev. Lett. 96, 105004 (2006)

[5] A. Pukhov and J. Meyer-ter-Vehn, Appl. Phys. B 74, 355 (2002).

[6] Leemans et al. Nature Phys. 2, 696 (2006)

[7] K. Nakamura et al., Phys. Plasmas 14, 056708 (2007)

[8] I. Blumenfeld et al. Nature 445, 741 (2007)

[9] P. Muggli, V. Yakimenko, M. Babzien, E. Kallos, K. P. Kusche Phys. Rev. Lett. 101, 54801 (2008)

[10] M. Minty, F. Zimmermann, Measurement and control of charged particle beams, Springer ISBN 9783540441878

[11] P.Antici et al. Coupling of Laser-Generated Electrons with Conventional Accelerator devices, to be published

[12] C. Lejeune and J. Aubert, Adv. Electron. Electron Phys. Suppl. A 13, 159 (1980)

[13] A. Mostacci et al. Review of Scientific Instruments 79, 013303 (2008) 
[14] T. Levato et al. AIP conf. proc. 1209, 59 (2010)

[15] C. Sears, A.Buck, K. Schmid, J. Mikhailova, F. Krausz, L. Veisz, Physical Review Special Topics Accelerator and Beams 13, 092803 (2010)

[16] E. Brunetti et al. PRL 105, 215007 (2010)

[17] N. Delerue Nuclear Instruments and Methods in Physics Research A 644 (2011) 110

[18] A. Mostacci, M. Bellaveglia, E. Chiadroni, A. Cianchi, M. Ferrario, D. Filippetto, G. Gatti, and C. Ronsivalle, Physical Review Special Topics - Accelerators and Beams 15, 082802 (2012)

[19] C. Thomas, N. Delerue and R. Bartolini, 2011 JINST 6 P07004 